\title{
Research on the Improvement Method of Music Education Level under the Background of AI Technology
}

\author{
Mao Qiusi (iD \\ Public Art Education Center, Henan Urban Construction College, Pingdingshan City, Henan, China \\ Correspondence should be addressed to Mao Qiusi; zxcv1231207@163.com
}

Received 7 December 2021; Revised 14 January 2022; Accepted 25 January 2022; Published 22 February 2022

Academic Editor: Hasan Ali Khattak

Copyright ( 2022 Mao Qiusi. This is an open access article distributed under the Creative Commons Attribution License, which permits unrestricted use, distribution, and reproduction in any medium, provided the original work is properly cited.

\begin{abstract}
To improve the demand pressure of traditional music on space, music equipment, and funds in the teaching mode, this study presents a music education model based on the model view controller (MVC) framework structure based on AI technology; the model optimizes traditional music education by intelligent adjustment of computational audio and three-frequency equalizer, through the integration of artificial intelligence and music education; seven feasible explorations are put forward; and its effects and the value and future development trend of artificial intelligence in music education were also analyzed. Subsequently, the bivariate $t$-test was used to check and accept the teaching effect of the implemented music education classroom, and teacher and parent satisfaction with music education in the AI background was compared. The results show that, compared with traditional teaching models, music education based on AI background has high satisfaction among students, teachers, and parents. This study lays a solid foundation for the future integration of music education and intelligent technology, and the investment of artificial intelligence can effectively expand the popularization of high-quality music education, thus expanding the level of universal music education in China, improving the national music literacy, and providing valuable development ideas for the research of artificial intelligence technology in other teaching fields.
\end{abstract}

\section{Introduction}

Deng mentioned in the article the source of artificial intelligence traced back to the 1950s, and artificial intelligence is a kind of using digital computer or using digital computer control machinery, imitates, replaces, and expands human behavior activities of intelligent technology and external environment, understands knowledge, and uses it to obtain the best scheme theory, scheme, technology, and application system [1]. With the rapid development of computer hardware and the continuous update of intelligent algorithms, artificial intelligence technology can realize audio recognition, image recognition, language recognition, natural language processing, and other functions and can be widely used in education, medical care, security, and many other fields. Wenhui said that this smart technology is likely to be applied to music education, it may have a big impact on traditional music education, and it is likely to cause a new trend of [2] in the field. Since China's Internet wave, in the late 20th century and the early 21 st century, the Internet has brought great changes to people's lives. Since the implementation of the strategy of rejuvenating the country through science and education, multimedia teaching has been gradually promoted and popularized, and the teaching methods in various disciplines, including music, have been innovated. Cao and Qi mentioned that AI technology was initially introduced into music classes in the 1960s, represented by simple music synthesizers, which store the timbre of various instruments and can play [3] at any time. With the rapid development of the Internet and the popularization of mobile clients, and the development of intelligent technology, technology has been upgraded, and many "products" are extended by this technology compared with before.

In recent years, artificial intelligence technology has been used to develop a considerable number of software applications, which all reflect the characteristics of "intelligent" and "humanized," to meet the needs of music teaching and interaction. The music classroom accepts and applies more 
efficient, more intelligent, and humanized synthetic musical instruments, and the upgrading of these musical instruments presents the characteristics of being more convenient, intelligent, and perfect. The application of scientific and technological means in the music field has laid the foundation for the creation of new works, while providing a new direction and teaching thinking for music educators.

\section{Application of AI Technology in Music Education}

2.1. Intelligent Model Construction of Music Education. Zhang and He said in the article that universities have used artificial intelligence platforms in discipline education and received well. To this, the application of artificial intelligence to music education has become an urgent problem to be solved at [4]. Fortunately, the application of artificial intelligence in music has long been the first. Its earliest application in music refers to the electronic synthesizer, which has certain intelligent functions. It can store and play the timbre of a variety of musical instruments, providing great convenience for integrated music. Sun Tianlin said that in the traditional music classroom, due to the limitation of teaching equipment and funds, teachers often use electronic synthesizers to show students the timbre of different musical instruments. This similar intelligent "behavior" also greatly improves the learning and practice efficiency of music education, which has been highly praised by relevant departments and has become the premise of the preparation of comprehensive teaching [5]. Music education is one of the most important ways of aesthetic education, which is the particularity of music education. Music class in addition to allowing students to appreciate excellent music works, master basic knowledge, and basic skills is more important to improve students' aesthetic taste. When combining AI technology with music education, the key elements of music should be integrated into the design of AI technology, as shown in Figure 1.

In Figure 1: showing: showing the students the classroom content; manageable: organizing the classroom teaching environment; accessible: teachers' understanding channels of teaching resources; real-time interactive: classroom interaction between teacher and student, and student interaction; testing: simulating music atmosphere for music teaching; computer accompaniment: realizing computer accompaniment using AI technology; music resource display: finding music resources quickly using AI technology; generate work: generating the works according to the input melody; The ONE: simulating online piano; interactive grading: classroom interaction between students and scoring each other's performance and statements; and Orb Composer: guiding students to perform in class.

The interactive score module can judge and advise the students' classroom performance (such as playing and singing) in the music class. Lu et al. mentioned that not only that but also students and students can interact to stimulate their enthusiasm for music learning in the form of a competition. The modules of computer accompaniment have powerful accompaniment functions and often appear in piano playing teaching and accompaniment courses [6]. He can automatically synthesize a harmony according to the melody given, and the students can be full of fun in this way, to enhance the students' music experience and creativity. Orb Composer is the first music intelligent composition software to use artificial intelligence technology, including six basic music style templates, which can form different music creation environments. It included pipes, strings, piano, electronics, pop rock, and ambient. Guo said that by choosing different tunes, rhythms, chords, and automatic generation steps, you can immediately generate a specified style of works, easy to use, and stimulate the enthusiasm of students [7]. The intelligent piano is a new piano teaching mode, containing a large number of high-quality resources at home and abroad, such as on-site teaching of piano playing, which can be watched online through the Internet and online competition. The module will be scored and evaluated according to the user's playing process.

Wang said in the study that intelligent classroom is a new classroom built based on the development of the Internet of things technology, cloud computing technology, and a variety of intelligent technologies. It is a typical materialized form of the intelligent learning environment. It consists of five dimensions, namely the SMART concept model, as shown in Figure 1: content display (the "Showing" in Figure 1), environmental control (the "Manageable" in Figure 1), resource acquisition (the "Accessible" in Figure 1), timely interaction (the "Real-time interactive" in Figure 1), and situational awareness (the "Testing" in Figure 1). Each dimension is used in the classroom, with a different emphasis, and these five parts form the AI music education [8].

2.2. Framework Required for Model Construction. The classroom module diagram of the AI music classroom introduced in this study is further constructed based on the smart classroom. The AI framework model used in this study is the model view controller (MVC) framework. One difficulty of the computer software design process in the system design is that it often fails to separate the view layer from the business layer, thus affecting the efficiency of the design work. Chou said that the MVC framework mode, because of its low coupling nature, itself has the separation of the view layer and the business layer. The designers do not need to recompile the whole model system and the controller code when filling in or changing the code. Similarly, changing an applied business process or business rule requires simply modifying the MVC model layer separated from the business layer, making such operations more simple; secondly, the service code of the MVC framework mode is very inclusive to the visiting view, sharing a model while using different access views, and can include all Web browsers and wireless browsers (WAP). It is well compatible with both modes, sharing a page when utilizing Web pages for access and when using internal links offline, and without interfering with [9].

For example, students can subscribe to the lessons they learn through mobile terminals such as a phone or computer, even when introduced by different hyperlinks, and the background handles their subscription commands. The data 


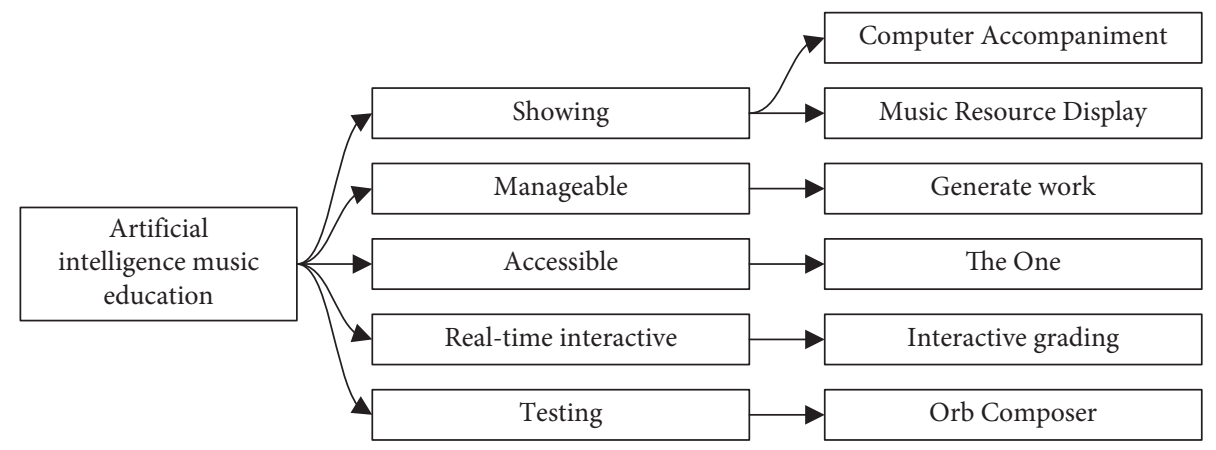

FIgURe 1: AI module distribution in music education.

returned by the model after processing are not formatted, so the same components can be used by different interfaces. Tian mentioned that again, the MVC framework mode has high subordinate efficiency and low life cycle, which can effectively reduce the technical content of the development and maintenance of the user interface, shorten the system development time, enable the background programmers, and interface programmers to focus on everything at hand, thus improving the efficiency of the framework deployment. In addition, after returning to the main page, again the previously clicked hyperlink is entered, and it has a memory mode and will automatically jump back to the last step of the last click, avoiding the loss of information caused by missing touch [10]. Finally, also due to the separation of the view layer and the business logic layer, the work of complex and costly system maintenance and background maintenance update modification coding becomes easier. The frame structure is as shown in Figure 2.

In Figure 2: view: the interface allows the user to interact with it; model: enterprise data and business rules; controller: to accept user input and call the model and views to complete the user's needs.

\subsection{Feasibility Analysis of AI Application in Music Education.} The feasibility analysis of the application of AI theory in music education can be understood and explained from the following seven different dimensions:

(1) Awake to: when teachers say the tone of a piece of music, students will have relevant thinking activities in independent practice, and their consciousness will also appear in some different states, such as reasoning, worry, reflection, empathy, and restatement. They can also integrate some conceptual and musical rational knowledge into the machine, provide "general" guidance for learners, and constantly add learners' thinking and consciousness, making it a multilevel thinking machine and become more conscious emotion.

(2) Cerebration: in the learning activities of music education, learners will instinctively respond to the normal operation of the body and brain, and have acquired reactions such as deep thinking and reflection; the higher values and thinking activities are entered, and self-conscious emotions are produced. When AI robots have these resources, they can become independent, self-aware entities.

(3) General knowledge: Liu and Cao said that some computer artificial intelligence with their own programs can only solve the special problems and the lack of common sense knowledge, which cannot achieve the expected goals of users, and reduce the quality and efficiency of artificial intelligence robots. To this end, AI machines should be given more common sense problems, so that they can realize common sense knowledge and reasoning, and are applied to the process of music teaching process, so that learners can learn how to manage their emotions. At the same time, because the same concert has different emotional and emotional effects on different people, notes and tunes can be used as a simplified "virtual" environment to refine the difference detector according to the difference network, help music learners obtain different music knowledge, memory, and thinking [11].

(4) Thought: the way humans think and solve problems is not limited to one kind, but can help music learners better break through the problems and difficulties in music learning. However, how to integrate these different ways of thinking, so that AI technology can effectively identify diverse ways of thinking, is a higher level that we want to explore, but also the future direction and problem type of AI intelligence application to music education.

(5) Intelligence: a computer can operate in different ways of thinking, and its functions enter the state that human beings are difficult to accurately evaluate under the continuous development of artificial intelligence technology.

(6) Oneself: after different time spans of time, artificial intelligence is expected to form computer self-concept and self-consciousness in the future, and artificial intelligence robots should also continue to seek and make progress on the road of self-concept and self-consciousness. 


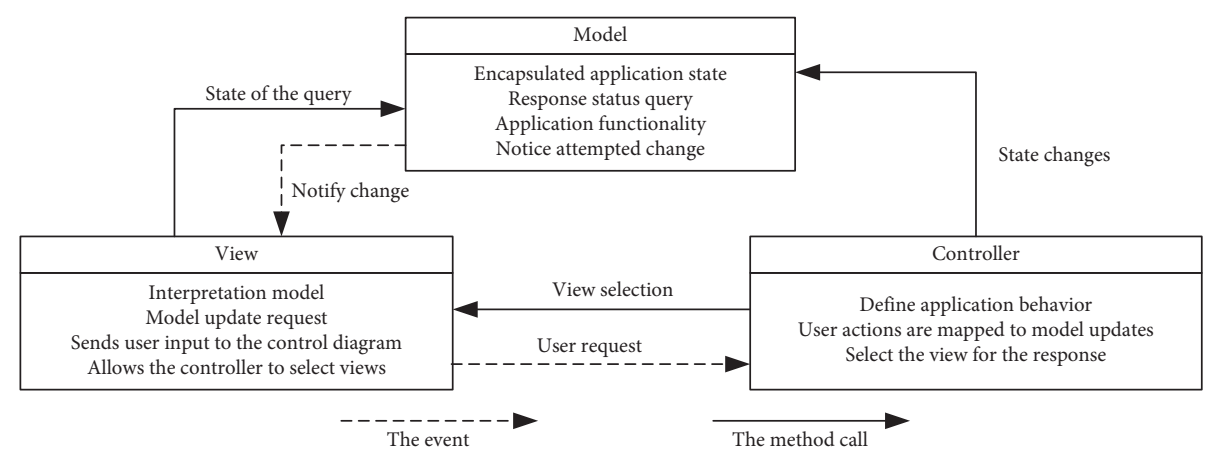

Figure 2: Internal framework of AI music model technology.

(7) Emotional interaction: Wang and Zheng said in the article of artificial intelligence technology in emotional computing function of breakthrough development and promotion [12] that it makes the computer can have similar to human communication, observation, understand or generate various emotions, can with human natural, cordial, vivid communication and interaction, if artificial intelligence technology is mature, will bring great convenience and subversion to the future music and education, and can make the music teaching process more vivid and interesting, in personalized.

In Figure 3: feasibility analysis: the feasibility exploration of the integration of music education and artificial intelligence; consciousness: integrating AI into consciousness; mental activity: making artificial intelligence to have thinking aesthetic thinking; common sense: the common sense contained in music inserts it into an AI program; thinking: jumping out of the inherent human stereotype and using technology to integrate music knowledge; smart: diversified ways of thinking; self: artificial intelligence has its own rules of thinking; emotional interaction: forming a human-computer emotional effect with human beings;

\section{The Role, Value, and Development of AI in Music Education}

3.1. Analysis of the Role of AI Technology in Music Education. The value and significance of AI technology in music education are embodied in the following aspects:

(1) Technology replaces human resources to realize the standardization of music education. Zhang Jun said that under the latest development of artificial intelligence technology, it has had a great impact and change on the employment market of teaching talents. Some automation jobs have replaced traditional workers, reduced market employment, and lost some employment opportunities. Therefore, more people can choose to join the teaching and training industry, and artificial intelligence has replaced part of the market of music teachers. Although it left the real teacher teaching, it improved the standardization of the overall music education market [13].
(2) Reduce the teaching pressure on teachers. Artificial intelligence teaching is different from the traditional teaching. Due to the particularity of the teaching content, compared with the acceptance of the teaching results, it is more important about how to mobilize the students' enthusiasm in class and their enthusiasm for music learning. Artificial intelligence technology happens to focus on solving this problem, and the curriculum atmosphere of AI teaching methods is more active, which also makes the connection between teachers and students even closer, and strengthens the interest of the classroom.

(3) Improve the students' music standards. Some students or parents who lack the basic knowledge of music lack a deeper learning music platform and learning opportunities and have no way to distinguish the music teaching level and ability of music teachers. Artificial intelligence technology can break through regional restrictions and use their excellent music resources and platforms to serve music learners.

\subsection{Application Value of AI Technology in Music Education}

(1) Improve the quality and efficiency of music teaching. Music education under the background of artificial intelligence technology makes use of its superior big data analysis technology and means of artificial intelligence to comprehensively analyze and understand the learning background, learning process, learning performance, and learning response of music learners, which provides a strong scientific basis for teachers to carry out music teaching and enables teachers to reasonably arrange the content and progress of music teaching. It improves the efficiency and quality of intelligent music teaching. For example, for students who do not express their emotions, music works that can fully express their emotions can be arranged. For students with public personality, they can choose music works with strong logical thinking and peaceful characteristic.

(2) Promote the improvement of students' learning efficiency. The learning of music requires a lot of technical hard practice and mental training. To 


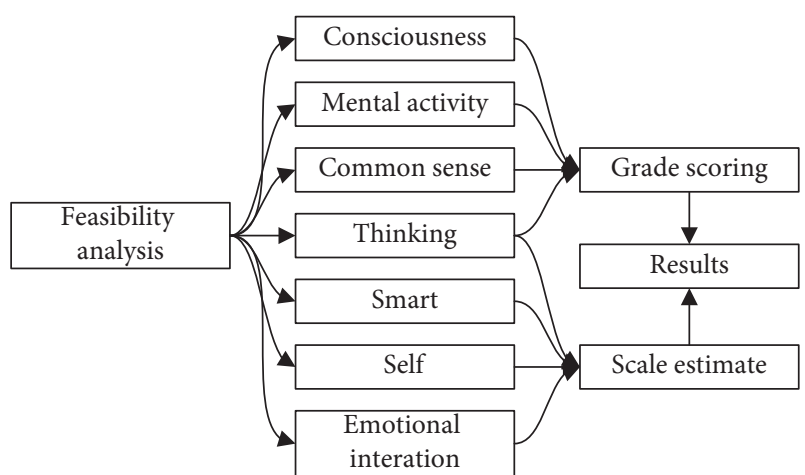

FIGURE 3: Feasibility analysis of AI application in music education.

change the boring state in music learning, artificial intelligence technology can be used to enhance students' interest in music learning, provide light and comfort for students' music learning, and promote the improvement of students' music learning efficiency.

(3) Integrate music learning resources. Xiaoyu mentioned that in the process of applying artificial intelligence technology in music education, music learning resources can be greatly integrated, and an extremely advanced music knowledge sharing system can be formed to provide convenience for teaching students in accordance with their aptitude [14].

\subsection{Development Trend of AI Technology in Music Education.} Wang said that artificial intelligence in the future society may understand the advantages and disadvantages of students in the process of music learning, has a large number of music resources, provides rich music resources for the music learners or enthusiasts, and helps students improve music education and learning efficiency faster [15].

Many years ago, in order to achieve a better effect of music teaching, we need to design large enough and place enough musical instruments to achieve the effect of sound field, which requires a lot of funds, space, and support of musical instruments. Otherwise, we can only use single and double channel music education, and the effect of music education is not ideal. In recent years, artificial intelligence has flourished in music education. Most music classrooms in colleges and universities use artificial intelligence technology for teaching. In the classroom, they use artificial intelligence to calculate the audio classroom so that students can act as live performances in the classroom and change the past teaching. When the funds and space are insufficient, musical instruments can only pass through mono and dual channels. The sound field is small and lacks defects. At the same time, music educators can effectively capture environmental noise through artificial intelligence-based computational audio technology and instantly simulate reverse sound waves, with only attempts to thicken music teacher walls or other physical layers to eliminate environmental noise. It can be seen that artificial intelligence technology greatly reduces the requirements for space and musical instrument equipment in music education. and you can try to intelligently adjust the music of the triband equalizer, so that everyone can hear the most suitable voice, so as to strengthen the effect of music education.

\section{Acceptance of the Actual Teaching Effect}

\subsection{Bivariate t-Calibration Algorithm}

4.1.1. T Checkout. The $t$-test, also known as Student's $t$-test, was mainly used for a normal distribution with a small sample content (e.g., $n<30$ ), and the overall standard deviation is unknown. The $t$-test is to infer the probability of differences occurring by the t-distribution theory and thus to compare whether the difference between the two averages is significant.

The t-test, also known as Student's $t$-test, applies mainly for normal distribution with small sample size, and the overall standard deviation is unknown. $\mathrm{t}$-Test such as formula (1).

The $\mathrm{t}$-value formula is as follows:

$$
\begin{aligned}
t & =\frac{|\bar{X}-\mu 0|}{S \bar{x}} \\
& =\frac{\bar{X}-\mu 0}{s / \sqrt{n}} .
\end{aligned}
$$

4.1.2. P Price. The $P$ value is the probability that results more extreme than the resulting sample observations appear when the null hypothesis is true. If the $P$ value is small, the probability of the null hypothesis is small, and the smaller the $P$ value, the more significant the results. In this study, the setting was considered as statistically significant between the two data groups when $T<10.000$ and $P<0.01$.

A coin was thrown 20 times with 14 fronts, and the probability of the event was plotted as a normal distribution, and then, the one-sided $P$ value of the sample was calculated as follows:

$$
\begin{aligned}
\operatorname{Prob}(14 \text { heads })+\operatorname{Prob}(15 \text { heads })+\cdots+\operatorname{Prob}(20 \text { heads }) & =\frac{1}{2^{20}}\left[\left(\frac{20}{14}\right)+\left(\frac{20}{15}\right)+\cdots+\left(\frac{20}{20}\right)\right] \\
& =\frac{60,460}{1,048,576} \approx 0.058 .
\end{aligned}
$$


4.2. Teachers' and Students' Satisfaction Survey with Teaching Methods. For the use effect of this teaching method, the actual use effect of schools in several provinces and cities is randomly checked and plotted in Table 1.

In Table 1: teacher satisfaction: teacher satisfaction with traditional teaching methods and AI teaching; student satisfaction: student satisfaction with traditional teaching methods and AI teaching; parent satisfaction: parent satisfaction with traditional teaching methods and AI teaching; traditional teaching methods: test satisfaction of teachers, students, and parents with teaching; AI teaching: test satisfaction of teachers, students, and parents under AI teaching;

After visual analysis of the data in Table 1, Figure 4 is obtained.

In Figure 4: the meanings of related terms are the same as those in Table 1.

It can be seen from Table 1 and Figure 4 that the AI teaching mode among teachers is widely praised, because the AI is helpful to teachers from all aspects, which greatly reduce the teaching burden of teachers, and the systematic platform teaching also increases the interest of the teaching classroom and makes the boring knowledge in the classroom no longer boring. Students are also very interested in this teaching mode. Different from the traditional classrooms, students can really speak freely in the classroom, integrate it into the classroom atmosphere, and their enthusiasm to obtain knowledge is also greatly improved. In contrast, parents have little response to the new teaching probably because artificial intelligence technology has not been widely introduced into parents' family model.

4.3. Survey of the Module Where Teachers and Students are Most Satisfied with the Teaching. This study conducted a sample survey of schools in Beijing and surrounding areas, issued 600 questionnaires, selected your three favorite modules and ranked them, and recorded the questionnaire results in Table 2.

In Figure 2: satisfaction 1: the most popular module among teachers, students, and parents; satisfaction 2: the second popular module among teachers, students, and parents; satisfaction 3: the third popular module among teachers, students, and parents; computer accompaniment: using AI technology to realize computer accompaniment; Orb Composer: guiding the performances that students show in class; The ONE online piano simulation; manageable: organizing the classroom teaching environment; accessible: teachers' understanding channels of teaching resources; real-time interactive: teacher-student interaction in the classroom, interaction between the students; testing: mocking the music atmosphere for music teaching.

As shown in Table 2, the most popular modules among teachers are resource acquisition, respectively, computer accompaniment, and environmental management. These three modules, respectively, reduce the burden of teachers from two aspects: pre-class lesson preparation and class teaching environment, shorten meaningless repetitive homework of teachers. A digital way can make teachers' work more efficient and convenient; the most popular student modules are focused on classes. The interactive link in the class makes the students and the teachers more closely connected with the classroom. Situational perception can make it easier for students to integrate into the music atmosphere in the classroom. Parents and teachers will pay more attention to students' performance in the classroom. Compared with extracurricular classroom education, parents pay more attention to the improvement of students' own knowledge in the classroom. The above table is made into a bar chart, and you can more intuitively see the love of the three modules, as shown in Figure 5.

\section{Future Development Expectations of Artificial Intelligence Technology in the Field of Music Education}

Contemporary social technology has been making continuous progress and innovation, and the development of artificial intelligence technology has also advanced by leaps and bounds recently, and the integration of artificial intelligence and music teaching is an inevitable situation. In the near future, perhaps AI can see going directly into the classroom, give students an immersive classroom experience, get real emotional feedback, or a real-time online music teacher who sees a 3D stereo projection just over the thousands of kilometers apart, and send cutting-edge, synchronous music teaching for children in remote areas. The $3 \mathrm{D}$ projection technology is used to generate real-time teaching images of music teachers, and teaching can be done, even thousands of miles apart. Education fairness is comprehensively promoted while maximizing the use of teachers. Technology is used to make more students. Even students in remote areas can learn about the humanities, and you can also enjoy the music education. Zhao said that on the other hand, the continuous progress of science and technology also gives music educators new hope and expectations. In the field of music education, the development direction of artificial intelligence should start from the simple technical level to perceptual thinking mining, because the number of music educators is limited, music education resources are also limited, so the investment in artificial intelligence can effectively expand the popularity of high-quality music education, so as to expand the level of universal music education in China, and improve the national music quality [16]. Secondly, in terms of the existing music creation and evaluation, it is also necessary to enrich the evaluation mechanism, optimize the evaluation structure, and optimize the algorithm to make the evaluation system more objective and real but without human touch. More random elements should be added in terms of music creation. Different from human emotions, the birth of technology can also fully explore the richness and diversity of music itself and provide more possibilities for music creation.

\section{Summary}

This study presents a music education model for AI technology based on the MVC framework structure, through the integration of AI with music education; seven feasibility 
TABLE 1: Satisfaction survey result table (\%).

\begin{tabular}{lccc}
\hline Grouping & Teacher satisfaction & Student satisfaction & Parents' satisfaction \\
\hline Traditional teaching methods & 64.6 & 57.5 & 78.5 \\
Artificial intelligence teaching & 83.2 & 70.6 & 82.1 \\
T & 1.647 & 1.937 & 0.647 \\
$P$ & 0.004 & 0.004 & 0.003 \\
\hline
\end{tabular}

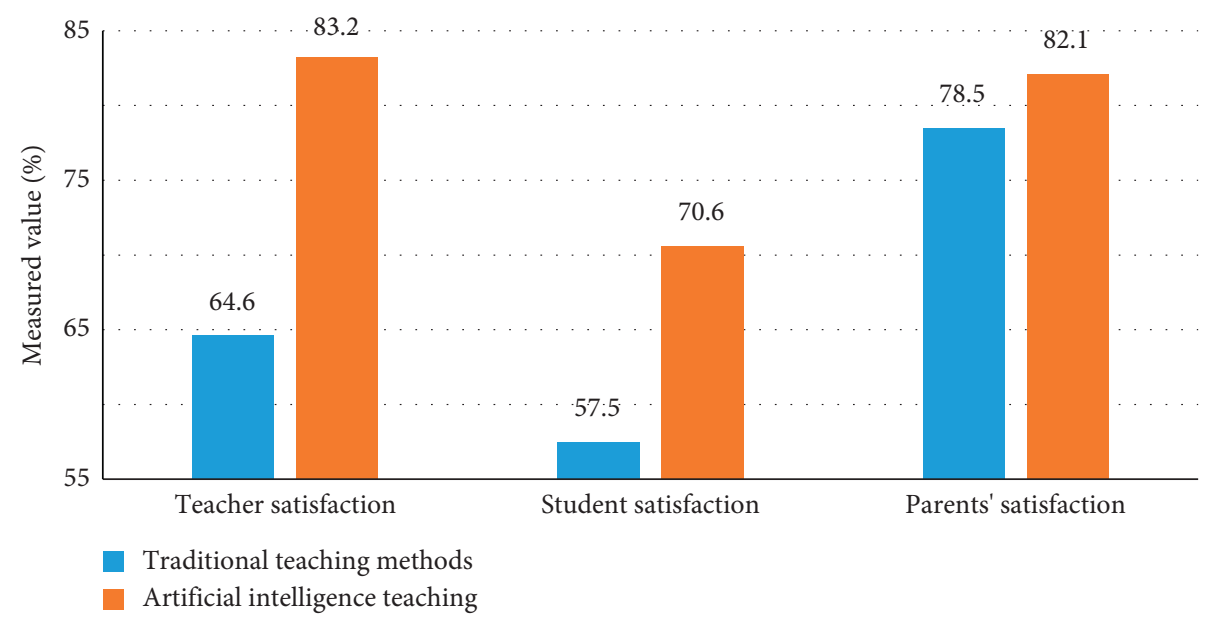

FIGURE 4: Satisfaction survey results.

TABLE 2: List of the most popular modules for teachers, students, and parents.

\begin{tabular}{lcccc}
\hline Grouping & Teacher & Student & Parents & Totality \\
\hline Satisfaction first & Accessible (92) & Real-time interactive (83) & The ONE (104) & Real-time interactive (141) \\
Satisfaction second & Computer accompaniment (65) & Testing (57) & Real-time interactive (58) & The ONE (137) \\
Satisfaction third & Manageable (19) & Orb Composer (16) & Testing (17) & Accessible (92) \\
\hline
\end{tabular}

Note: the numbers in parentheses are the specific number of questionnaires.

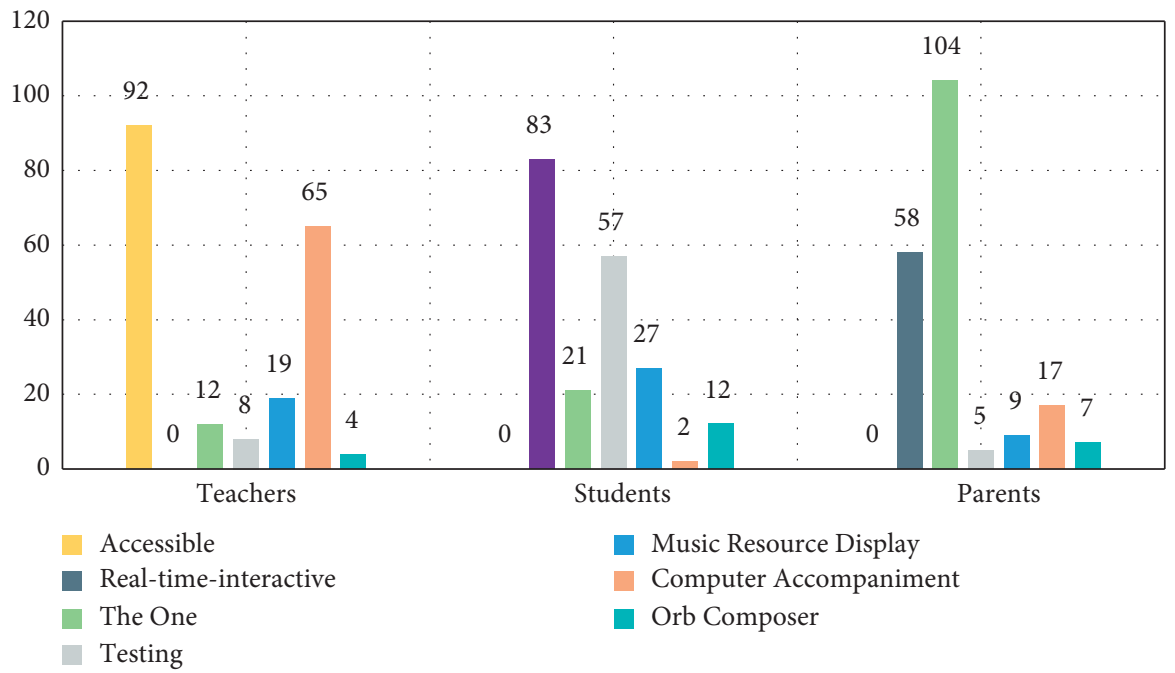

FIGURE 5: Distribution diagram of the modules most favored by students, teachers, and parents.

explorations of the role of AI in music education are proposed and analyzed; and the value and future development trend of artificial intelligence in music education are analyzed. Subsequently, the bivariate $t$-test was used to accept the $t$-effect, comparing teachers' and students' and parents' satisfaction with music education in the background of AI. The results showed that compared with the traditional teaching models, music education in the background of AI 
has high satisfaction among students, teaching, and parents. This study lays a solid foundation for the future integration of music education and intelligent technology, it has expanded the level of music education in China and improved the national music literacy, and it provides valuable development ideas for the research of AI technology in other teaching fields.

\section{Data Availability}

The data underlying the results presented in the study are included within the manuscript.

\section{Conflicts of Interest}

The author declares that there are no conflicts of interest.

\section{Authors' Contributions}

The author has read the manuscript and approved to submit it to your journal.

\section{References}

[1] X. Deng, "Innovative application of artificial intelligence in the self-diagnosis and treatment of psychological problems and health education in universities," Journal of Jilin Medical College, vol. 42, no. 06, pp. 427-428, 2021.

[2] Wenhui, "Thoughts on the interaction between AI technology and music education," Sichuan Drama, no. 09, pp. 170-172, 2021.

[3] R. Cao and M. Qi, "On the development of artificial intelligence and ideological and political education course," Educational observation, vol. 10, no. 41, pp. 53-56, 2021.

[4] M. Zhang and H. He, "Modernization of Higher Education Courses in the Intelligent Era: Motivation and Path," Xinyang Normal University Journal (Philosophy and Social Sciences, vol. 41, 2021.

[5] T. Sun, "Man-technology relationship game and construction in AI education is - - from reverse domestication to technical mediation," Open Education Research, vol. 27, no. 6, pp. 37-43, 2021.

[6] G. Lu, K. Xie, Q. Liu, C. Zhang, and S. Yu, "Analysis of classroom teaching behavior based on AI engine," Open Education Research, vol. 27, no. 06, pp. 97-107, 2021.

[7] Q. Guo, "Existing Problems and Coping Strategies in Higher Vocational College Music Education," Theatre House, vol. 41, no. 34, pp. 179-180, 2021.

[8] S. Wang, "Exploration and Thinking on Online Music Teaching," Theatre House, no. 33, pp. 176-177, 2021.

[9] J. Chou, "The design and implementation of the Japanese teaching management system based on the MVC architecture," Scientific and technological innovation and productivity, no. 10, pp. 148-150, 2021.

[10] Tian, "Design and implementation of university multimedia management system based on MVC framework," Mechanical design, vol. 38, no. 09, pp. 123-124, 2021.

[11] L. Wen and H. Cao, "Exploration on dance teaching mode under the new trend of AI + education," Contemporary music, no. 11, pp. 169-171, 2021.

[12] Y. Wang and Y. Zheng, "Intelligent education products: build a new ecology of intelligent education based on AIoT," Open Education Research, vol. 27, no. 06, pp. 15-23, 2021.
[13] J. Zhang and Y. Zhao, "The research and application of the interactive teaching music intelligence system based on artificial intelligence," Microcomputer Applications, vol. 37, no. 4, pp. 45-48, 2021.

[14] H. Xiaoyu, "The current situation and future development of music education software in teaching," Contemporary Music, no. 1, pp. 154-157, 2021.

[15] Z. Wang, "The application of artificial intelligence in music and its development strategy," Northern Music, no. 22, pp. 211-213, 2020.

[16] Yu Zhao, "Research on the Application of AI in Music Education," Theatre House, no. 27, pp. 57-58, 2020. 